\title{
Comparison of Ising Model and Potts Model on Non-Local Directed Small-World Networks
}

\author{
M. A. Sumour ${ }^{*}$, M. Kh. Srour11, S. M. Baraka', M. A. Radwan'1, R. J. Khozondar1, M. M. Shabat ${ }^{3}$ \\ ${ }^{1}$ Physics Department, Faculty of Science, Al-Aqsa University, Gaza, Gaza Strip, Palestine \\ ${ }^{2}$ National Institute of Aerospace, Hampton, VA, USA \\ ${ }^{3}$ Department of Physics, Faculty of Science, Islamic University of Gaza, Gaza Strip, Palestine, Palestine \\ Email: *ma.sumoor@alaqsa.edu.ps
}

How to cite this paper: Sumour, M.A., Srour, M.Kh., Baraka, S.M., Radwan, M.A., Khozondar, R.J. and Shabat, M.M. (2020) Comparison of Ising Model and Potts Model on Non-Local Directed Small-World Networks. Journal of Applied Mathematics and Physics, 8, 1031-1038.

https://doi.org/10.4236/jamp.2020.86080

Received: May 3, 2020

Accepted: June 2, 2020

Published: June 5, 2020

Copyright $\odot 2020$ by author(s) and Scientific Research Publishing Inc. This work is licensed under the Creative Commons Attribution International License (CC BY 4.0).

http://creativecommons.org/licenses/by/4.0/

\begin{abstract}
Further to the investigation of the critical properties of the Potts model with $q$ $=3$ and 8 states in one dimension (1D) on directed small-world networks reported by Aquino and Lima, which presents, in fact, a second-order phase transition with a new set of critical exponents, in addition to what was reported in Sumour and Lima in studying Ising model on non-local directed small-world for several values of probability $0<P<1$. In this paper the behavior of two models discussed previously, will be re-examined to study differences between their behavior on directed small-world networks for networks of different values of probability $P=0.1,0.2,0.3,0.4$ and 0.5 with different lattice sizes $L=10,20,30,40$, and 50 to compare between the important physical variables between Ising and Potts models on the directed small-world networks. We found in our paper that is a phase transitions in both Ising and Potts models depending essentially on the probability $P$.
\end{abstract}

\section{Keywords}

Ising Model, Potts Model, Directed Small World, Probability, Magnetization, Susceptibility

\section{Introduction}

Networks of coupled dynamics systems have been used to numerically model many self-organizing systems, such as biological oscillators, neural networks, spatial games and genetic control networks [1] and the references therein. With resourceful computing fascicles using modeling techniques such as Monte Carlo codes [2], the simulation of the small-world networks become doable [3] [4] showed that the Ising model and Potts Model on a small-world (SW) network presents a phase transition well defined at a finite temperature. [2] studied the 
Potts model with $q=3$ and 4 states on directed small-world networks (DSWN) as a function of temperature. [5] covered the Potts model for $q=4$ and had found a first-order phase transition for values of the rewiring probability $P=0.1$ and $P=0.9$ that agree with the [6].

In the directed small world networks, each node is randomly reconnected with $\mathrm{n}$ edges with probability $P$. In this paper, we employed values of probability as $(P=0.01,0.2,0.3,0.4,0.5)$, and the lattice size $(L=10,20,30,40,50)$, and adopted values from both Ising and Potts model in purpose of comparison between them.

According to reference [7], Potts model in two-dimension (2D) presents a phase transition at finite temperature $T$, for any number of states $q>1$, and as in reference [8]. Ising model on non-local directed small-world lattices has a second-order phase transition with new critical exponents dependent on $p(0<p$ $<1)$.

Most of all of researches in Ising and Potts models studied the magnetization, susceptibility, fourth-order Binder Cumulant, and energy. All of these parameters were studied as a function of temperature. There parameters are determined and analyzed for both models [4] [8] to study the similarities and differences between them. The behavior of these models on the directed small-world networks for networks is covered.

\section{Model and Simulations}

In our simulations, values and shapes are adopted as in [9] these parameters are doable. We start our simulations for different values of $L$ and $P$ as follows: $L=10$, 20 , and 40 and probability $P=0.1,0.2,0.3,0.4, \& 0.5$, with magnetization as a function of temperature (0 to 4 ) in Kelvin. As a case study $P=0.3$, was chosen to be plotted herein. We shall now define all parameters on the two models as defined in statistical physics as listed below:

\subsection{Ising Model on Non-Local Directed Small-World Networks}

For Ising model we considered here the molar magnetization $m$, as $m=\sum_{i} \sigma_{i} / L$, where $\sigma_{i}$ is a spin variable at each node of the network, and $L$ is the length of a linear chain where.

$$
m=[\langle|m|\rangle]_{a v},
$$

the susceptibility

$$
\chi(T)=\frac{L}{T}\left[\left\langle m^{2}\right\rangle-\langle m\rangle^{2}\right]_{a v}
$$

and the fourth-order Binder Cumulant

$$
U_{4}(T)=1-\left[\frac{\left\langle m^{4}\right\rangle}{3\langle|m|\rangle^{2}}\right]_{a v}
$$

In the above equations $\langle\ldots\rangle$ stand for thermodynamic averages and $[\ldots]_{a v}$ 
for averages over different realizations. To calculate the exponents of these models, we apply the finite-size scaling (FSS) theory [10]. We expect, for large system sizes, an asymptotic FSS behavior of the form:

$$
\begin{aligned}
& m=L^{-\beta / v} f_{m}(x)[1+\cdots], \\
& \chi=L^{\gamma / v} f_{\chi}(x)[1+\cdots],
\end{aligned}
$$

where $\beta$ and $\gamma$ are the usual critical exponents, and $f_{i}(x)$ are FSS functions with

$$
x=\left(T-T_{c}\right) L^{1 / v},
$$

being the scaling variable. The dots in the brackets $[1+\cdots]$ indicate corrections to scaling terms. We calculated the error bars from the fluctuations among the different realizations. Therefore, from the size dependence of $M$ and $\chi$, we obtain the exponents ratios $\beta / v$ and $\gamma / v$, respectively. The susceptibility at its maximum also scales as $L^{\gamma / v}$

Moreover, the value of $q$ for which $\chi$ has a maximum, $T_{c}^{\chi_{\max }}=T_{c}(L)$ scales with the lattice size as:

$$
T_{C}(L)=T_{C}+b L^{-1 / v}
$$

where $b$ is a non-universal constant.

The correlation length exponent $1 / v$ can be estimated from Equation (7).

\subsection{Potts Model on Non-Local Directed Small-World Networs}

For Potts model as in reference [8], we use the energy per spin, $e=E / N$, and the magnetization per spin, $m=M / N$ with $M=\left(q \max \left[n_{i}\right]-N\right) /(q-1)$, were evaluated. Here $n_{i} \leq N$ denotes the number of spins with "orientation" $i=1, \cdots, q$. From the energy measurements, we can compute the average energy, specific heat, and energetic cumulant.

$$
\begin{gathered}
u(T)=[\langle e\rangle]_{a v} \\
C(T)=\frac{N}{T^{2}}\left(\left[\left\langle e^{2}\right\rangle\right]_{a v}-[\langle e\rangle]_{a v}^{2}\right) \\
B_{e}(T)=1-\frac{\left[\left\langle e^{4}\right\rangle\right]_{a v}}{3\left[\left\langle e^{2}\right\rangle\right]_{a v}^{2}}
\end{gathered}
$$

Similarly, we can derive from the magnetization measurements the average magnetization, the susceptibility, and the fourth-order magnetic Cumulant,

$$
\begin{gathered}
m(T)=[\langle|m|\rangle]_{a v} \\
\left\langle\chi(T)=\frac{L}{T}\left[\left\langle m^{2}\right\rangle\right]_{a v}-[\langle|m|\rangle]_{a v}^{2}\right| \\
U_{4}(T)=1-\left[\frac{\left\langle m^{4}\right\rangle}{3\langle|m|\rangle^{2}}\right]_{a v}
\end{gathered}
$$


In order to calculate the exponents for this model, we applied the finite-size scaling (FSS) theory, and for large system sizes we got [9]

$$
\begin{gathered}
C=C_{\text {reg }}(T)+L^{\alpha / v} f_{C}(x)[1+\cdots], \\
{[\langle|m|\rangle]_{a v}=L^{-\beta / v} f_{m}(x)[1+\cdots],} \\
\chi=\chi_{\text {reg }}(T)+L^{\gamma / v} f_{\chi}(x)[1+\cdots],
\end{gathered}
$$

where $C_{\text {reg }}(T)$ and $\chi_{\text {reg }}(T)$ are regular temperature dependent background terms. $v, \alpha, \beta$, and $\gamma$ are the usual critical exponents, and $f_{i}(x)$, with $i=C, m, \chi, p$, are FSS functions with

$$
x=(K-K c) L^{1 / v}
$$

being the scaling variable, and $K=J / k_{B} T$. We calculated the error bars from the fluctuations among the different realizations. Note that these errors contain both the average thermodynamic error for a given realization, and the theoretical variance for infinitely accurate thermodynamic averages which are caused by the variation of the quenched random geometry of the networks.

For both Ising and Potts models from the magnetization, we can investigate other measures such as the average magnetization, susceptibility and the fourth-order Binder Cumulant, for probability $p=0.5$ to 1 . and for Potts model the phase transition is always first order. For probability $p=0.5$ to 1 and for Ising, the code doesn't work well. And for both models and $p=1$ the networks are not a small world, they are a complex network.

\section{Results and Discussion}

When all plots and probability values are done, the magnetization behavior shows a very small change. On the other hand, when the size of $L=10,20$, and 40 the changes in Figure 1 show that the magnetization of Potts model (left plots in Figure 1) decayed faster than Ising model (right plot in Figure 1), the values of magnetization varies slowly with temperatures after 2.1 kelvin.

Also, we determined the "flatness" of the curves of Potts and Ising model as $(-0.76)$, and $(-2.87)$ respectively, and we find that Ising and Potts model illustrates a continuous phase transition and the decay behavior of magnetization which agrees with magnetization universality.

Moreover, we plot the susceptibility as function of temperature ( 0 to 4$)$ in Klein for $L=10,20$, and 40 and probability $=0.1,0.2,0.3,0.4,0.5$, a case study of is presented as $P=0.3$, and we take the $\log$ scale for the $y$-axis because there is variation in values. Figure 2 shows a shift in the peak as the lattice size $(L)$ increases, the peak of $L=40$ is taking the maximum peak of all values, and for Ising model the average peak is at 2.5 while for Potts model is at 1.1, and the shape of the peak for Potts model more sharp than the Ising model.

Figure 3 shows the behavior of energy versus temperature ( 0 to 4 ) for $L=10$, 20 , and 40 and probability $=0.1,0.2,0.3,0.4,0.5, P=0.3$ is again our sample plot, we can see a typical behavior attesting for a first-order phase transition in the 
range of all $P$ values, and we see a variation at the beginning point of Ising model at $\mathrm{x}$-axis $=1.39363$, but Potts model at 0.575685 and intersection at the point $(x$ $=2.8783, y=-0.844846$ and we noticed that the energy of Potts model after temperature of 2 Kelvin becomes more stable than the Ising model after $T=2.5$ Kelvin.

Figure 4 shows the Fourth-order Binder Cumulant (U4) as a function of Temperature ( 0 to 4$)$ for several lattice sizes $L=10,20$, and 40 and probability $=$ $0.1,0.2,0.3,0.4, \& 0.5$ and we plot as a sample $P=0.5$. Also, we notice a continuous phase transition for all values. We notice that for Potts model at $L=40$ there is a sharp peak concave down at 1.9 temperature.

The actual values of the magnetization for each $L$ to Ising and Potts model for the probability $P=0.3$ as shown in Table 1 , from these values we get Figure 5 which shows the difference between the values of magnetization of Ising and Potts model with lattice size for sample probability $P=0.3$. Also, we can clearly see that the exponent is, within the errors, independent of $P$, in agreement with universality ideas [8].

In Table 1 and from our simulation that used huge computing time and space, the magnetization values for both Ising and Potts Model for three Lattices sizes $(10,20$ and 40$)$ is tabulated in Table 1.

Figure 5 shows that the values of magnetization of Ising model is larger and varies with the magnetization of the Potts model, but the values of magnetization of Potts model is stable.

Table 1. The values of magnetization for Ising and Potts model with different lattice sizes $(L)$.

\begin{tabular}{ccc}
\hline$L$ & Magnetization for Ising & Magnetization for Potts \\
\hline 10 & 0.90832949499980631 & 0.11721970000000800 \\
20 & 0.91423680999999635 & $5.83670812499994876 \mathrm{E}-002$ \\
40 & 0.914494221875002 & $2.92451515624998562 \mathrm{E}-002$ \\
\hline
\end{tabular}

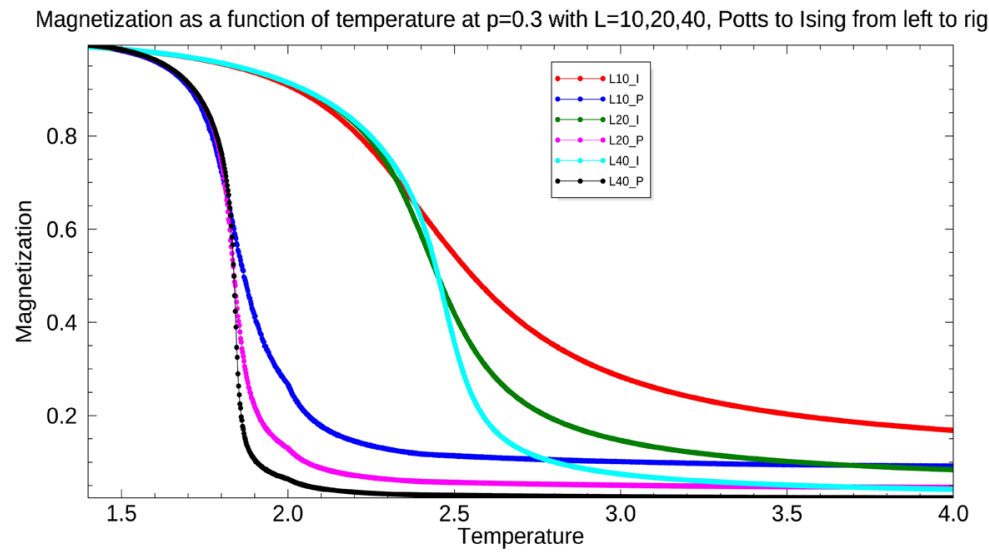

Figure 1. Magnetization versus temperature for sizes $L=10,20$, and 40 with rewiring probability $p=0.3$. 


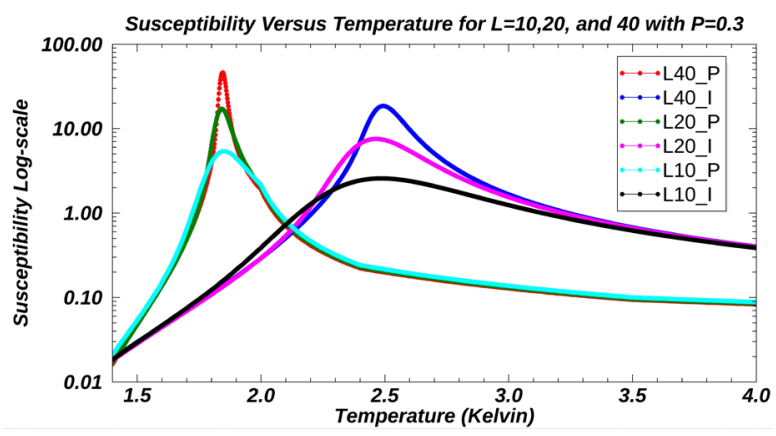

Figure 2. Susceptibility versus temperature for sizes $L=10$, 20 , and 40 with rewiring probability $p=0.3$.

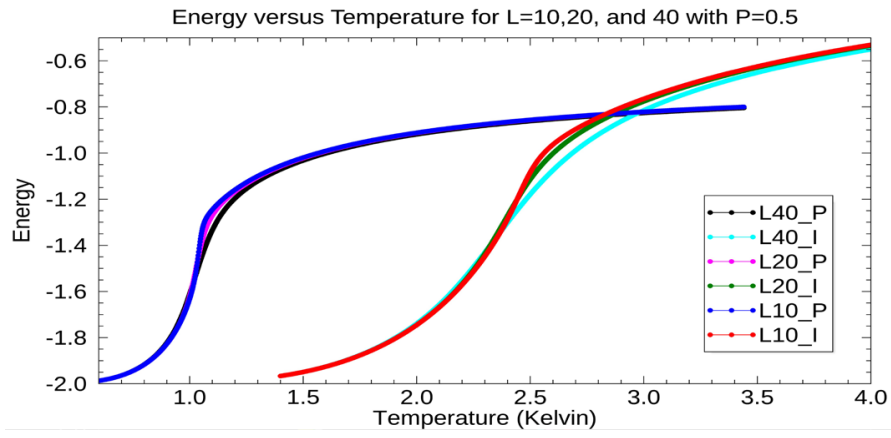

Figure 3. Shows the behavior of energy versus temperature (0 to 4 ) for $L=10,20$, and 40 and probability $=0.1,0.2,0.3,0.4,0.5, P=0.3$.

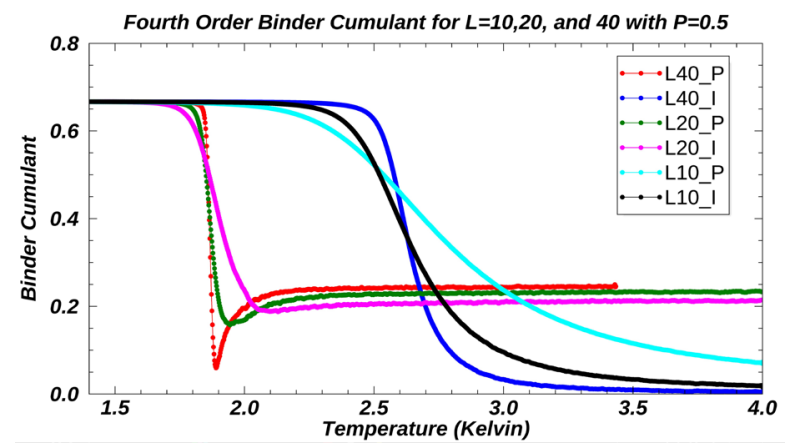

Figure 4. Fourth-order Binder Cumulant (U4) temperature for sizes $L=10,20$, and 40 with rewiring probability $p=0.5$.

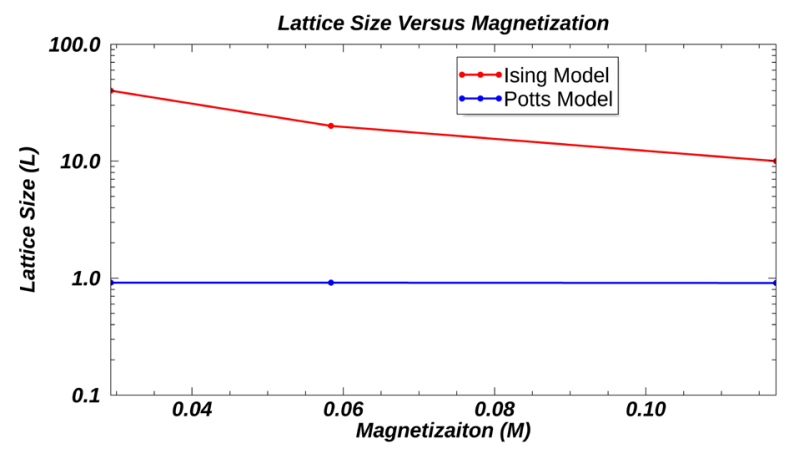

Figure 5. Lattice size $(L)$ versus Magnetization $(M)$ for sizes $L$ $=10,20$, and 40 with rewiring probability $p=0.3$. 


\section{Conclusion}

The Potts and Ising models are studied for different Lattice sizes and different probability on non-local directed small-world networks, including long-range interaction between spins depending on the probability $P$. We found a phase transitions in both Ising and Potts models depending essentially on the probability $P$. This behavior is the influence of long-range interactions that occur in the presence of $P$ directed bonds and also the number of the Potts model states on the directed SW network. The change in the universality of these models can be described as the influence of directed non local interactions that occur with the presence of $P$ directed bonds [11].

\section{Acknowledgements}

S. Baraka thanks the IAP-CNRS, France, for compiling the Ising Model on their supercomputing facilities. And authors would like to express thanks to the University of Natural Resources and Life Sciences (BOKU) at Vienna in Austria for giving our access to their supercomputer to do our simulation of our work.

\section{Conflicts of Interest}

The authors declare no conflicts of interest regarding the publication of this paper.

\section{References}

[1] Watts, D.J. and Strogatz, S.H. (1998) Collective Dynamics of "Small-World" Networks. Nature, 393, 440. https://doi.org/10.1038/30918

[2] Da Silva, P.R.O., Lima, F.W.S. and Costa Filho, R.N. (2013) Potts Model with $q=3$ and 4 States on Directed Small-World Network. Computer Physics Communications, 184, 2746-2750. https://doi.org/10.1016/j.cpc.2013.07.020

[3] Pȩkalski, A. (2001) Ising Model on a Small World Network. Physical Review E, 64, 057104. https://doi.org/10.1103/PhysRevE.64.057104

[4] Hong, H., Choi, M.-Y. and Kim, B.J. (2002) Synchronization on Small-World Networks. Physical Review E, 65, 026139. https://doi.org/10.1103/PhysRevE.65.026139

[5] Lima, F.W.S. and Plascak, J.A. (2013) Critical Behavior of the Ising and Blume-Capel Models on Directed Two-Dimensional Small-World Networks. The European Physical Journal B, 86, 300. https://doi.org/10.1140/epjb/e2013-40165-5

[6] Janke, W. and Weigel, M. (2004) Harris-Luck Criterion for Random Lattices. Physical Review B, 69, 144208. https://doi.org/10.1103/PhysRevB.69.144208

[7] Lima, F. (2019) Potts Model on Directed Complex Networks. Physics \& Astronomy International Journal, 3, 156-157. https://doi.org/10.15406/paij.2019.03.00175

[8] Sumour, M.A. and Lima, F.W. (2019) Critical Behavior of the Ising Model on Non-Local Directed Small-World Networks. IOSR Journal of Applied Physics (IOSR-JAP), 11, 1-7.

[9] Aquino, É.O., Lima, F.W.S., Araújo, A.D. and Costa Filho, R.N. (2018) Potts Model in One-Dimension on Directed Small-World Networks. Journal of Statistical Physics, 171, 1112-1121. https://doi.org/10.1007/s10955-018-2046-5

[10] Baxter, R.J. (1982) The Inversion Relation Method for Some Two-Dimensional Exactly Solved Models in Lattice Statistics. Journal of Statistical Physics, 28, 1-41. 
https://doi.org/10.1007/BF01011621

[11] Ferraz, C.H.A. and Lima, J.L.S. (2017) Three-State Potts Model on Non-Local Directed Small-World Lattices. Physica A: Statistical Mechanics and Its Applications, 484, 488-498. https://doi.org/10.1016/j.physa.2017.05.016 